\title{
Diacronie
}

Studi di Storia Contemporanea

$N^{\circ} 33,1 \mid 2018$

Guerra e pace

\section{Rolf Petri (a cura di), Balcani, Europa. Violenza, politica, memoria}

\section{Eric Gobetti}

\section{(2) OpenEdition \\ Journals}

Edizione digitale

URL: http://journals.openedition.org/diacronie/7531

DOI: $10.4000 /$ diacronie.7531

ISSN: 2038-0925

Editore

Association culturelle Diacronie

Notizia bibliografica digitale

Eric Gobetti, «Rolf Petri (a cura di), Balcani, Europa. Violenza, politica, memoria », Diacronie [Online], N

33, 1 | 2018, documento 11, Messo online il 29 mars 2018, consultato il 24 septembre 2020. URL:

http://journals.openedition.org/diacronie/7531 ; DOI : https://doi.org/10.4000/diacronie.7531 


\section{Diacronie}

Studi di Storia Contemporanea

\section{$33,1 / 2018$}

Guerra e pace: declinazioni politiche, sociali e culturali del conflitto in età contemporanea

\section{RECENSIONE: Rolf PETRI (a cura di), Balcani, Europa. Violenza, politica, memoria, Torino, Giappichelli, 2017, 175 pp.}

\section{A cura di Eric GOBETTI}

Per citare questo articolo:

GOBETTI, Eric, «RECENSIONE: Rolf PETRI (a cura di), Balcani, Europa. Violenza, politica, memoria, Torino, Giappichelli, 2017, 175 pp. », Diacronie. Studi di Storia Contemporanea : Guerra e pace: declinazioni politiche, sociali e culturali del conflitto in età contemporanea, 33, 1/2018, 29/03/2018,

URL: < http://www.studistorici.com/2018/03/29/gobetti_numero_33/ >

Diacronie Studi di Storia Contemporanea $\rightarrow$ http://www.diacronie.it

Rivista storica online. Uscita trimestrale.

redazione.diacronie@hotmail.it

Comitato di direzione: Naor Ben-Yehoyada - João Fábio Bertonha - Christopher Denis-Delacour - Maximiliano Fuentes Codera Anders Granås Kjøstvedt - John Paul Newman - Deborah Paci - Niccolò Pianciola - Spyridon Ploumidis - Wilko Graf Von Hardenberg

Comitato di redazione: Jacopo Bassi - Luca Bufarale - Gianluca Canè - Luca G. Manenti - Fausto Pietrancosta - Alessandro Salvador - Matteo Tomasoni - Luca Zuccolo 


\title{
11/ RECENSIONE: Rolf PETRI (a cura di), Balcani, Europa. Violenza, politica, memoria, Torino, Giappichelli, 2017, 175 pp.
}

\author{
A cura di Eric GOBETTI
}

Non è la prima volta che un autore o una serie di autori affrontano il tema complesso dei rapporti fra Balcani, violenza e politica. Il tema è stato però declinato solo da pochi anni in una accezione diversa da quella che ha dominato l'immaginario collettivo dell'Europa nel secolo scorso. Divenuti quasi sinonimo di primitiva attitudine al conflitto nel corso del lungo Ottocento, in una fase di affrancamento dall'Impero Ottomano che è durata di fatto cento anni, i Balcani hanno mantenuto e rafforzato questa identificazione nel corso del secolo successivo. I conflitti che hanno messo uno contro l'altro gli stati nazionali sorti con la caduta degli imperi multinazionali; le guerre mondiali, con il loro corollario di violenze contro i civili; i regimi dittatoriali fra le due guerre e la cappa oppressiva dei paesi d'oltre cortina durante la Guerra Fredda; sono tutti elementi che hanno rafforzato questo stereotipo. Uno stereotipo sostanzialmente razzista, che attribuiva al mondo balcanico una particolare attitudine alla violenza e all'odio etnico o religioso.

Ormai da tempo, però, i migliori studiosi dell'area si sono affrancati dall'immaginario "balcanista" che vorrebbe quest'area geografica particolarmente portata al conflitto: una parte d'Europa «non ancora pienamente civile, moderna, democratica, umanista, europeizzata, occidentalizzata, intraprendente, ingegnosa, economicamente sviluppata» ${ }^{1}$. Il volume curato da Rolf Petri, docente di storia contemporanea all'Università Ca' Foscari di Venezia, aggiunge un significativo tassello a questo filone di studi, tra i quali spicca l'importante lavoro di Stefano Petrungaro, Balcani: una storia di violenza? edito nel $2012^{2}$.

I due saggi introduttivi, a firma dello stesso Petri e di Stefano Petrungaro, ne delineano le prospettive essenziali, sottolineando quanto sia necessario assumere una prospettiva che non

\footnotetext{
${ }^{1}$ PETRI, Rolf, Balcani. Teleologia di una regione, in ID. (a cura di), Balcani, Europa. Violenza, politica, memoria, Torino, Giappichelli, 2017, pp. 1-22, p. 10.

2 PETRUNGARO, Stefano, Balcani. Una storia di violenza?, Roma, Carocci, 2012.
} 
guardi in maniera teleologica alla storia dei Balcani. In particolare, la puntuale comparazione con il resto d'Europa consente di includere legittimamente i fenomeni storici che hanno attraversato quest'area nel corso del Novecento in un percorso comune a tutto il continente. Il doloroso affrancamento dagli imperi multinazionali, il difficile percorso per la creazione di stati nazionali omogenei, la diffusione di una cultura politica autoritaria fra le due guerre, le violenze ideologiche e nazionaliste nel corso dei conflitti mondiali, sono tutti fenomeni che accomunano gran parte d'Europa. Così anche l'autoidentificazione nella categoria dei popoli civili e la necessità di porre «l'Altro e il nemico su un più basso gradino dello sviluppo umano: loro i barbari, noi i civili» ${ }^{3}$, sarebbero processi che si riscontrano in tutto il continente europeo nel corso del Novecento. Petrungaro in particolare fa notare come proprio la comparazione con altre realtà europee e mondiali consenta di relativizzare quella supposta "cieca ferocia" che sarebbe tipica dei popoli balcanici, identificando piuttosto nella «situazione etno-demografica, lascito delle precedenti esperienze imperiali» ${ }^{4}$ e nel ricorso ad una sorta di "eugenetica sociale" tipica dei processi di State-building, le ragioni contingenti di alcuni picchi di violenza che hanno caratterizzato i Balcani nel corso del Novecento.

La raccolta di saggi che compone il volume riesce a dare un quadro molto ampio dei rapporti fra violenza politica e creazione degli stati nazionali nel Sudest Europa nell'arco di più di un secolo, dalla seconda metà dell'Ottocento alla fine del Novecento, offrendo al lettore uno scenario per niente uniforme e molto complesso. Partendo dal triste presupposto che «il XX secolo è stato forse il secolo più violento della storia umana ${ }^{5}$, saggi di storici politici, studiosi di storia delle idee ed esperti di politiche della memoria si alternano per offrire differenti punti di vista su fenomeni storici che riguardano diversi paesi dell'area: Grecia, Bulgaria, Romania, Jugoslavia e paesi eredi.

Il testo di apertura, a firma di Armando Pitassio, offre un interessante resoconto dell'uso della violenza politica nel contesto bulgaro a cavallo dei due secoli XIX e XX, e del suo rapporto ambiguo con la regione macedone. In questo caso è la complessa costruzione dello Stato-nazione a comportare un uso massiccio del terrorismo e della violenza ai danni delle popolazioni considerate non omologabili (i 150.000 turchi espulsi dopo l'indipendenza) o di quelle non del tutto integrate (macedoni o bulgari fedeli al Patriarcato di Costantinopoli).

Il saggio di Francesco Zavatti, quasi consequenziale in termini temporali, mette l'accento sulla violenza come ideologia politica nel legionarismo rumeno fra le due guerre. Per questo

3 PETRI, Rolf, Balcani. Teleologia di una regione, in ID. (a cura di), op. cit., pp. 1-22, p. 21.

${ }^{4}$ PETRUNGARO, Stefano, Tra particolare e generale. Sulla violenza nei Balcani, in PETRI, Rolf (a cura di), op. cit., pp. 23-36, p. 27.

${ }^{5}$ VOGLIS, Polymeris, Violenza e cambiamento sociale nella Grecia occupata. Riflessioni sulle guerre del Novecento, in PETRI, Rolf (a cura di), op. cit., pp. 95-112, p. 95. 
movimento, inizialmente «quasi privo di un programma politico vero e proprio [...], il culto della morte, della violenza e della guerra erano una rilettura in chiave politica dei vangeli» ${ }^{6}$.

Milovan Pisarri presenta un'attenta analisi di come evolve in un secolo la memoria delle vittime civili dei diversi conflitti che attraversano la Serbia nel corso del Novecento. È interessante dunque notare come ogni nuovo Stato adotti una politica della memoria «differente, contrastante rispetto a quella precedente» ${ }^{7}$. A proposito della seconda guerra mondiale colpisce il capovolgimento di prospettiva fra l'epoca socialista, quando l'accento era posto sulle vittime della guerra contro gli invasori, e l'attuale Stato indipendente, che ha istituito un "Giorno della memoria delle vittime serbe della seconda guerra mondiale", in larga parte vittime dei massacri commessi dagli ustascia croati. Si tratta in definitiva di una "nazionalizzazione della memoria" con l'intento di «accorpare astoricamente tutte le vittime serbe del XX secolo in un'unica narrazione nazionale» ${ }^{8}$ che include anche le guerre degli anni Novanta, dove i serbi possono essere presentati sempre solo come vittime e mai come colpevoli.

Polymeris Voglis evidenzia nel suo saggio la militarizzazione dell'intera società greca durante l'occupazione italo-tedesco-bulgara nella seconda guerra mondiale. Coerentemente con il resto d'Europa, anche in Grecia il conflitto è stato caratterizzato dalla progressiva scomparsa di una «netta linea di demarcazione tra soldati e civili»" ${ }^{9}$, connessa a una serie di fenomeni concatenati tra loro: l'occupazione militare, la resistenza, la repressione, la guerra civile. In un contesto del genere, l'autore sottolinea quanto sia sfumato l'elemento della "volontarietà" nell'adesione ad uno dei campi in lotta e quanto la costrizione fisica e le condizioni economiche disperate abbiano giocato un ruolo determinante in tali "scelte".

Partendo dalle emanazioni del diritto umanitario dopo la prima guerra mondiale, Luca Baldissara giunge ad analizzare le implicazioni in termini giuridici e psico-sociali del tribunale ad hoc per i crimini nella ex Jugoslavia. Questo ultimo caso evidenzia in maniera paradigmatica «l'inestinguibile contraddizione» tra la «funzione di deterrenza del crimine di guerra» e la «giustizia politica», ovvero «l'attribuzione al vincitore della facoltà di giudicare» ${ }^{10}$. Questa contraddizione è indiscutibilmente aggravata da due fenomeni che caratterizzano i conflitti del Novecento: il discrimine sempre più sottile fra militare e civile - ad esempio in contesti di guerra partigiana o di conflitto civile - e l'identificazione sempre più esplicita del civile (in quanto appartenente ad una comunità identificata in senso razziale, linguistico, religioso) come target strategico, obiettivo finale del conflitto stesso.

\footnotetext{
${ }^{6}$ ZAVATTI, Francesco, La violenza nell'ideologia del legionarismo romeno (1923-1941), in PETRI, Rolf (a cura di), op. cit., pp. 57-74, pp. 62-63.

${ }^{7}$ PISARRI, Milovan, La memoria delle vittime civili in Serbia, in PETRI, Rolf (a cura di), op. cit., pp. 75-94, p. 78.

${ }^{8}$ Ibidem, p. 92.

${ }^{9}$ VOGLIS, Polymeris, op. cit., in PETRI, Rolf (a cura di), op. cit., pp. 95-112, p. 97.

${ }^{10}$ BALDISSARA, Luca, Dal punto di vista del diritto. Violenza bellica e punizione dei crimini di guerra, in PETRI, Rolf (a cura di), op. cit., pp. 113-130, p. 122.
} 
Infine l'ultimo saggio, firmato da Maurizio Cermel, mostra come la creazione di stati-nazione dopo l'implosione della Jugoslavia negli anni Novanta del Novecento abbia messo le minoranze nazionali in una pericolosa condizione di minorità anche giuridica. Sia la costituzione croata del 1990 che la legge approvata dall'Assemblea nazionale della repubblica di Serbia nel 2006 identificano lo stato in senso etnico, distinguendo tra il popolo di riferimento e le minoranze nazionali, alle quali viene «garantita l'uguaglianza» (costituzione croata) e verso le quali è «vietata ogni forma di discriminazione» (legge serba), ma che appartengono indiscutibilmente ad una categoria altra rispetto ai veri e propri cittadini dello stato.

Rimane in definitiva, oltre ad una vastissima bibliografia e a molte nuove e utili informazioni, la sensazione generale che i Balcani non possano più essere considerati, nemmeno in senso storico, un corpo estraneo al continente. Al contrario, leggendo questi testi, si direbbe che ne siano la parte più viva, quella che con maggiore virulenza ne ha espresso l'anima profonda, mostrando tutte le ambiguità e i pericoli di logiche esclusiviste, autoritarie, violente che sempre più tornano in auge. I Balcani, in definitiva, sono lo specchio deformante d'Europa, uno specchio nel quale i dettagli possono confondere, ma aiutano anche a comprendere la natura profonda del continente in cui viviamo. 


\section{L'AUTORE}

Eric GOBETTI (Torino, 1973) è uno storico free-lance, studioso di Seconda guerra mondiale e della Jugoslavia nel Novecento. È curatore di diversi volumi e autore di tre monografie: Dittatore per caso. Un piccolo duce protetto dall'Italia fascista (Napoli, L'ancora del Mediterraneo, 2001), sul movimento croato Ustascia negli anni Trenta; L'occupazione allegra. Italiani in Jugoslavia 1941-1943 (Roma, Carocci, 2007); Alleati del nemico. L'occupazione italiana in Jugoslavia (Roma-Bari, Laterza, 2013). Ha inoltre pubblicato il diario-reportage Nema problema! Jugoslavie, 10 anni di viaggi (Torino, Miraggi edizioni, 2011) e negli ultimi anni organizza viaggi di turismo storico nei paesi della ex Jugoslavia. È apparso più volte sul canale televisivo RaiStoria, realizzando tra l'altro nel 2013 la trasmissione in tre puntate La Divisione Garibaldi. Nel 2015 ha prodotto il suo primo documentario storico: Partizani. La Resistenza italiana in Montenegro. Nel 2017 ha portato a termine un progetto sul centenario dell'attentato di Sarajevo, con la pubblicazione del libro Sarajevo Rewind. Cent'anni d'Europa (Torino, Miraggi edizioni, 2017) e l'uscita del secondo film Sarajevo Rewind 2014>1914 (con Simone Malavolti).

URL: < http://www.studistorici.com/progett/autori/\#Gobetti > 